\title{
The Impact of Social Media on College Mental Health During the COVID-19 Pandemic: a Multinational Review of the Existing Literature
}

\author{
Jessica M. Haddad ${ }^{1,2}$. Christina Macenski ${ }^{1,2}$. Alison Mosier-Mills ${ }^{2} \cdot$ Alice Hibara $^{5} \cdot$ Katherine Kester $^{2}$. \\ Marguerite Schneider ${ }^{2,3} \cdot$ Rachel C. Conrad ${ }^{1,2}$. Cindy H. Liu ${ }^{1,2,4}$
}

Accepted: 27 August 2021 / Published online: 6 October 2021

( ) The Author(s), under exclusive licence to Springer Science+Business Media, LLC, part of Springer Nature 2021

\begin{abstract}
Purpose of Review During the COVID-19 pandemic, both social media use and rates of anxiety and depression among college students have increased significantly. This begs the question, what is the relationship between social media use and college student mental health during the COVID-19 pandemic?

Recent Findings Prior studies have found mixed results regarding the relationship between social media use and college student mental health. This relationship has become increasingly complex during the COVID-19 pandemic. We found that excessive or problematic social media use during the COVID-19 pandemic was correlated with worse mental health outcomes that could be mitigated by dialectical thinking, optimism, mindfulness, and cognitive reappraisal.

Summary The COVID-19 pandemic acts as a moderator by strengthening the relationship between social media use and mental health. Future studies should consider the impact of social media on college student mental health and concentrate on intervention initiatives to ensure the psychological well-being of college students during a global pandemic outbreak.
\end{abstract}

Keywords COVID-19 $\cdot$ Pandemic $\cdot$ College students $\cdot$ Mental health $\cdot$ Social media $\cdot$ Literature review

\section{Introduction}

The novel coronavirus (SARS-CoV-2) and its corresponding disease (COVID-19) have had tremendous impacts on mental health across the globe [1-5]. College students have been among the most strongly affected by the COVID-19

This article is part of the Topical Collection on Complex MedicalPsychiatric Issues

Jessica M. Haddad

jhaddad@bwh.harvard.edu

1 Department of Psychiatry, Brigham and Women's Hospital, 75 Francis St., 221 Longwood Avenue, Boston, MA 02115, USA

2 Harvard Medical School, 25 Shattuck St, Boston, MA 02115, USA

3 Boston Children's Hospital, 300 Longwood Avenue, Boston, MA 02115, USA

4 Department of Newborn Medicine, Brigham and Women's Hospital, 75 Francis St., 221 Longwood Avenue, Boston, MA 02115, USA

5 Harvard College, 86 Brattle Street, Cambridge, MA 02138, USA pandemic [6-8]. According to the Household Pulse Study, rates of anxiety and depression in college-age individuals have grown rapidly during the pandemic $[9 \bullet \bullet]$. In addition to depression and anxiety, college students have experienced worsening rates of low self-esteem, loneliness, stress, substance misuse, and suicidality [10-17]. This may be because college students are encountering unique stressors including interruptions in their education, technological concerns about online learning, hindrance of physical activity and extracurricular activities, social isolation, lack of college belongingness, decreased family income, financial concerns threatening their future education, and uncertainty about future employment [18-20].

Social media use has increased among almost all cohorts during the COVID-19 era [21], but younger people have been particularly affected $[22,23]$. Generation Z, defined as the generation born after 1996, constitutes the majority of current college students. College students today are "digital natives"; they were raised during the age of digital technology and therefore have been familiar with the internet and social media from an early age. Although college students' social media use is ubiquitous at baseline, it has intensified 
during the COVID-19 pandemic [24•, 25]. An increased level of engagement with the internet was required for educational and other purposes due to the pandemic, which potentially predisposed college students to a range of experiences that may be either positive or negative.

Prior studies regarding the relationship between social media use and college student mental health have had mixed findings. Some have shown that excessive screen time during the pandemic has a negative impact on mental health $[26 \bullet, 27]$. Social media use in particular appears to be linked to depression and secondary trauma during the pandemic [28•, 29•]. Psychological distress from social media may be partially explained by extensive COVID- 19 coverage [30, 31], in part due to the algorithmic structure of social media programming [32•]. Although social media may exacerbate COVID-19-related stress [33•], social media use has skyrocketed during the pandemic [21], and at least one study found an inverse relationship between social media use and

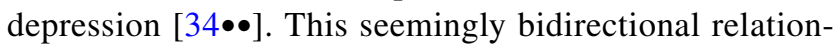
ship is complex and indicates that social media use during the pandemic has both positive and negative properties (Table 1).

To the best of our knowledge, this is the first literature review to explore the impact of social media on the mental health of college students during the COVID-19 pandemic. As such, the authors seek to answer the following question: What is the role of social media on college student mental health during the COVID-19 pandemic?

\section{Methods}

\section{Search Methodology}

This article is a narrative review of the existing literature regarding the impact of social media on college student mental health during the COVID-19 pandemic. However, our search initially began by reviewing all literature regarding college student mental health in the setting of the pandemic to determine predominant themes. A search of the PubMed electronic database and Google Scholar was undertaken using the following search terms: Coronavirus, COVID-19, 2019-ncov, SARS-cov-2, Mental illness, Mental health, Psychiatry, Psychology, Anxiety, Depression, College, and University. All combinations of these keywords were included. As such, the PubMed search used the following query:

((Coronavirus [Title/Abstract]) OR (COVID-19[Title/ Abstract]) OR (2019-ncov [Title/Abstract]) OR (SARS-cov2[Title/Abstract])) AND ((Mental illness [Title/Abstract]) OR (Mental health [Title/Abstract]) OR (Psychiatry [Title/ Abstract]) OR (Psychology [Title/Abstract]) OR (Anxiety [Title/Abstract]) OR (Depression [Title/Abstract])) AND ((College [Title/Abstract]) OR (University [Title/ Abstract])).

Articles published between the beginning of the COVID19 pandemic and January 3rd, 2021 (date of last search) were screened for inclusion and exclusion criteria.

Table 1 Pros and cons of social media during the COVID-19 pandemic

\begin{tabular}{|c|c|}
\hline Pros of social media & Cons of social media \\
\hline 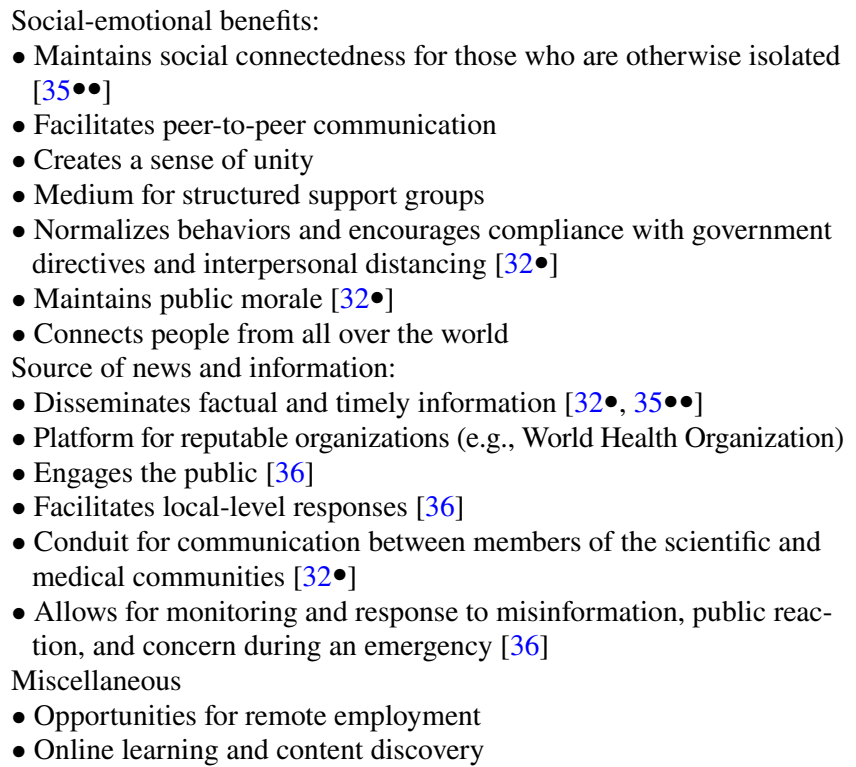 & 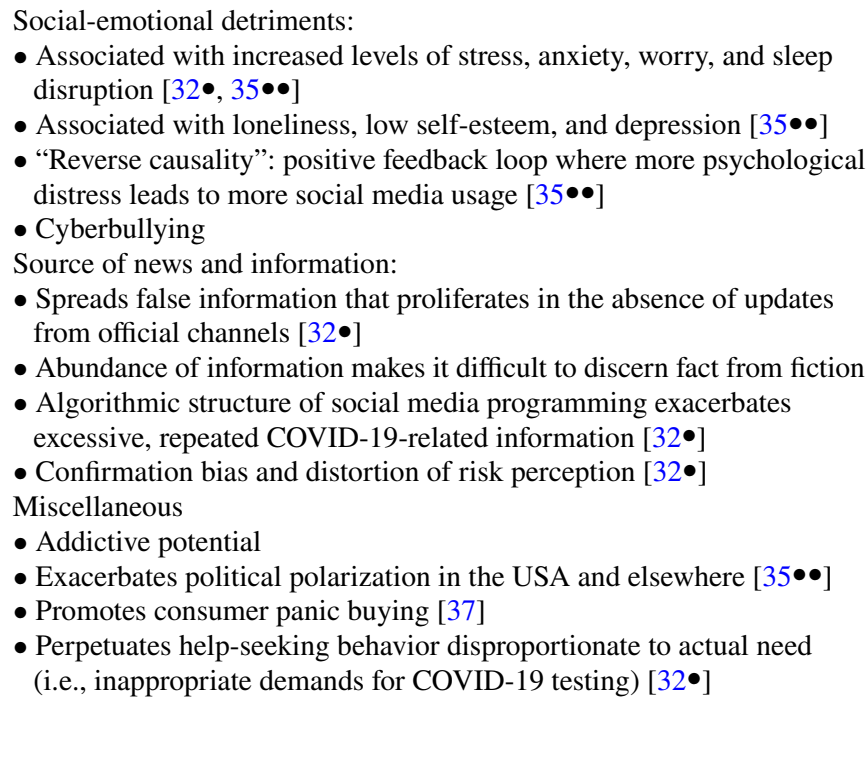 \\
\hline
\end{tabular}




\section{Inclusion and Exclusion Criteria}

Inclusion criteria for incorporation into the narrative review included the following: (1) observational studies (i.e., noninterventional), (2) original research, (3) English language, (4) studies that evaluated depression among college students using validated instruments, (5) studies that evaluated anxiety among college students using validated instruments, (6) included data on social media, (7) included COVID-specific data, and (8) provided demographic data or other predictors of mental illness.

Exclusion criteria included the following: (1) unrelated research works, (2) duplicate studies, (3) case reports or nonoriginal research (e.g., letters to the editor and review studies), (4) abstracts without full text, (5) studies not exclusive to college students, and (6) studies that did not have data on social media.

\section{Definition of Social Media and Thematic Analysis of Selected Articles}

After inclusion and exclusion criteria were applied, remaining articles were examined. The COVID-19 pandemic increased reliance on social media for all forms of communication during a time where in-person contact was inherently dangerous. Despite much focus on social media in the scientific literature, a reliable definition has yet to emerge, likely due to its rapidly evolving nature. For the purposes of this review, we define social media broadly as interactive, digitally mediated social networks.

Two broad themes were ultimately identified in the literature regarding the impact of social media on college student mental health: (1) mental health problems related to social media use during the COVID-19 pandemic and (2) emotionregulation strategies in relation to social media use and mental health during the COVID-19 pandemic.

\section{Results}

\section{Study Eligibility}

A total of 360 articles were retrieved. Articles were manually screened by title and abstract while considering the inclusion and exclusion criteria, which resulted in removal of 281 articles. Data were then extracted from the remaining 89 articles, and 83 additional articles were excluded thereafter (per exclusion criteria) (Fig. 1). The remaining 6 articles were all original observational studies of college mental health related to social media use during the COVID19 pandemic. Details and characteristics of these articles are provided in Table 2. Of note, several studies did not meet full criteria for inclusion, but included some relevant data and are thus briefly discussed herein.
Fig. 1 Eligibility flow diagram of study selection

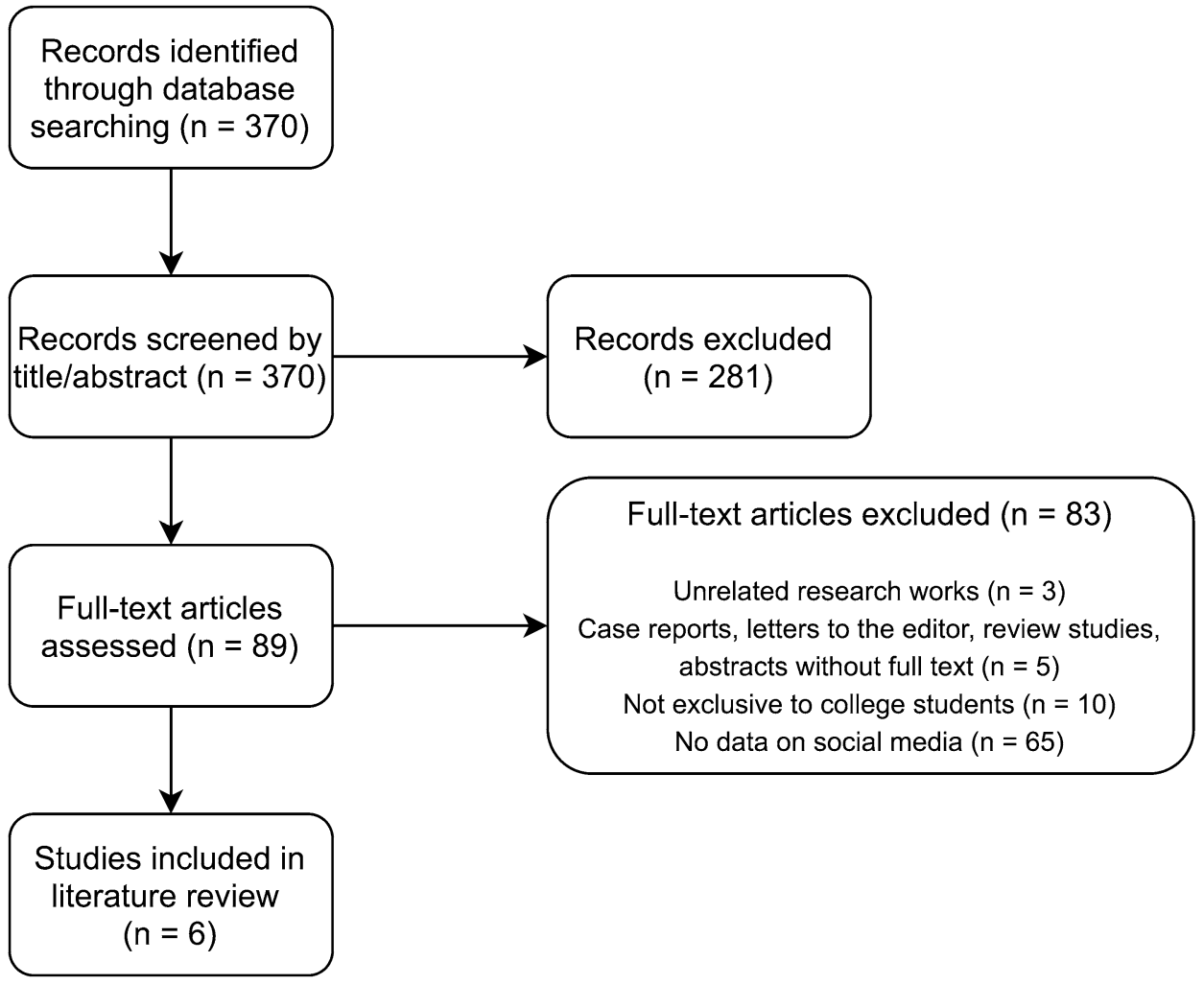




\section{The Relations Between Social Media Use and College Student Mental Health Symptoms During the COVID-19 Pandemic}

Three out of six articles studied the potential effects of social media on college student mental health during the COVID19 pandemic. Overall, study results were mixed in that social media use had negative, positive, and null effects on mental health among community samples of college students. Each article is summarized below.

One study from China collected data at two stages of the pandemic: shortly after the start of the pandemic (T1) and 1 month later (T2) [39••]. Compared to T1, social media use during T2 was significantly lower $\left(\chi^{2} / t=158.18, p<0.001\right)$, indicating more frequent social media use during the start of the pandemic. Heavy social media use ( $>3 \mathrm{~h} /$ day) at T1 was found to be a significant predictor of acute stress $(p<0.001)$ and anxiety symptoms $(p<0.001)$, but not of depressive symptoms $(p<0.05)$. Students who reported higher levels of acute stress during $\mathrm{T} 1$ were more likely to report higher levels of depression, anxiety, and worry about contracting COVID-19 during T2. The authors conclude that heavy social media use is associated with increased rates of depression and anxiety. These results suggest that social media may have a negative influence on short- and long-term mental health.

A second large-scale Chinese cross-sectional study [34••] also found that social media was associated with negative mental health measures. Social media use (average time spent per day accessing COVID-19-related content) was significantly associated with secondary trauma $(b=0.18$, $p<0.001)$, depressive symptoms $(b=0.11, p=0.019)$, and anxiety symptoms $(b=0.12, p=0.014)$. Additionally, high social media use was positively associated with depressive symptoms when college students reported higher levels of COVID-19 stress (assessed using a 10-item COVID stressor scale, e.g., witnessed a close death, lack of medical care, and lack of disinfectants), an interaction that the low COVID-19 stressor group did not exhibit. This suggests that COVID-19 stress moderates the relationship between social media use and depression. COVID-19 stress was not found to moderate the relationship between social media and anxiety or secondary trauma. Furthermore, negative affect (assessed using the Positive and Negative Affect Schedule) accounted for the relationship between social media use and mental health.

Finally, one study from the Netherlands [42••] did not find that social media use (e.g., overall habits and total number of friends on social media platforms) had a negative impact on mental health outcomes during the COVID19 pandemic. In fact, researchers found that social media use was negatively associated with depressive symptoms ( $r=-0.156 ; p<0.05$ ), suggesting that social media use and depression have an inverse relationship. There was no statistically significant association between social media use and anxiety $(r=0.078 ; p>0.5)$, loneliness $(r=-0.116$; $p>0.5$ ), or the COVID-19 stressor (impact of events on student life; $r=0.112 ; p>0.5$ ).

Given the mixed results, the relationship between social media use and college student mental health during the COVID-19 pandemic appears to be complicated and may be explained by moderating factors such as COVID-19 stress [34••]. There may be other mediating and moderating factors that affect this relationship, which could explain why social media use and depression were inversely related in a study that accounted for social media intensity and meaning $[42 \bullet \bullet]$. It may be that social media use attenuates depressive symptoms in college students who have meaningful relationships through social media (i.e., sense of belonging to an online community), who use it as a form of coping, or who use it as a replacement for maladaptive behaviors.

\section{Literature Addressing Emotion-Regulation Strategies in Relation to Social Media Use and Mental Health During the COVID-19 Pandemic}

A few studies explored whether cognitive thought patterns or emotional regulation strategies could affect the relationship between social media use and mental health during the COVID-19 pandemic. They will be described below.

One study in Italy [43•] considered the association between different thinking styles and the levels of distress felt by university students during lockdown. University students were invited to join a free online emotional support service that included secure messaging and video chats with providers. Before joining the service, students completed several validated instruments to assess depression and anxiety symptomatology, along with narrative questions regarding their emotions/worries. The investigators asked participants to keep a digital diary, which showed that students with "all or nothing" thinking — seeing the world only in terms of extremes - were five times more likely than other students to report posttraumatic symptomatology. Presence of this maladaptive thinking pattern may predict the severity of stress reactions to traumatic and/or life-changing events. Unsurprisingly, students with an "optimistic" thinking style reported fewer depressive, anxious, or posttraumatic symptoms, suggesting that optimism can be an important resource for adjustment to stressful situations.

Investigators in China [40••] surveyed college students on social media use, mindfulness, rumination, and measures of psychological distress during COVID-19. They found that social media exposure was associated with rumination and that students with ruminations were more likely to have negative cognitions and higher levels of psychological distress. They also found that mindfulness was a protective factor that attenuated the adverse effect of social media on mental 


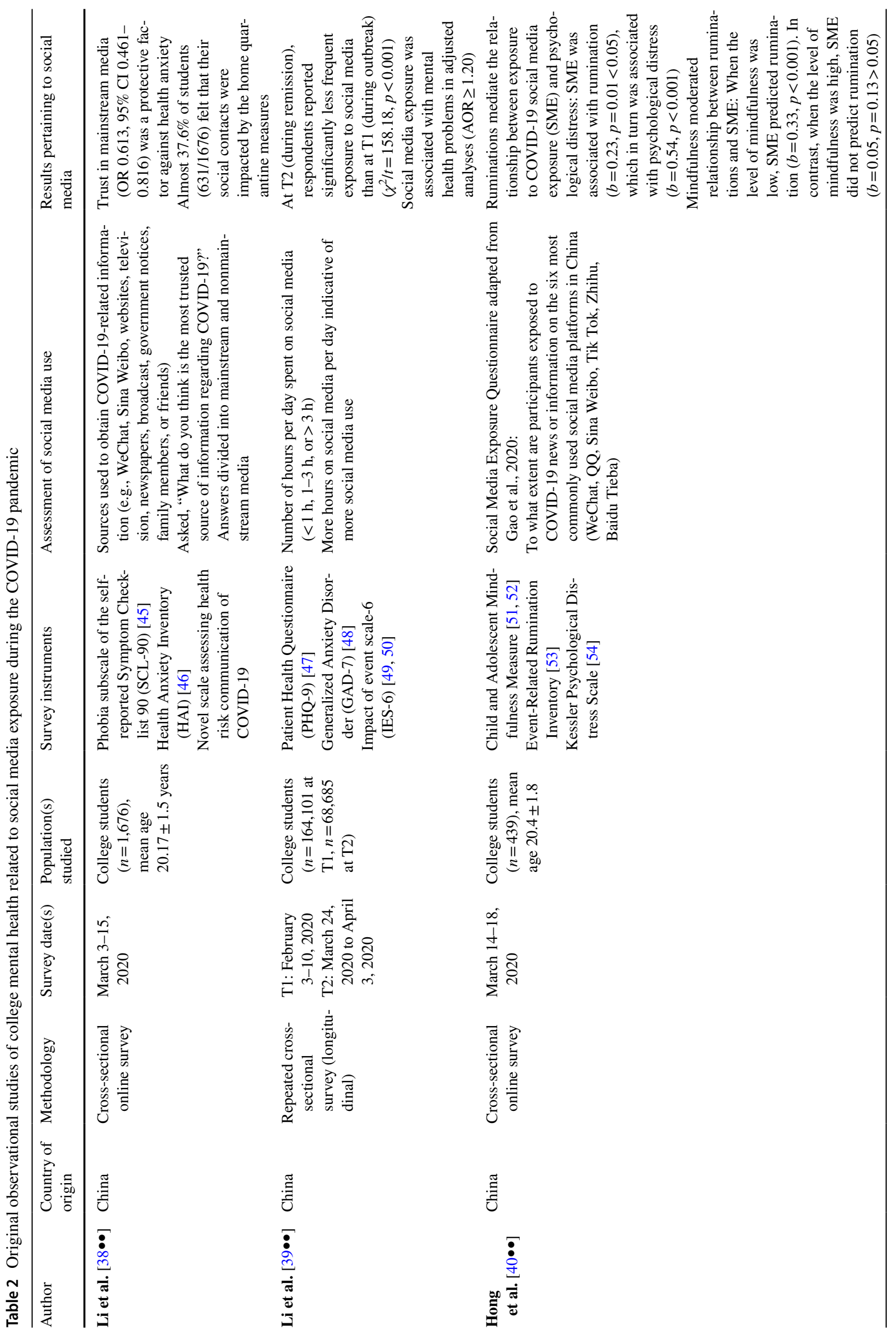




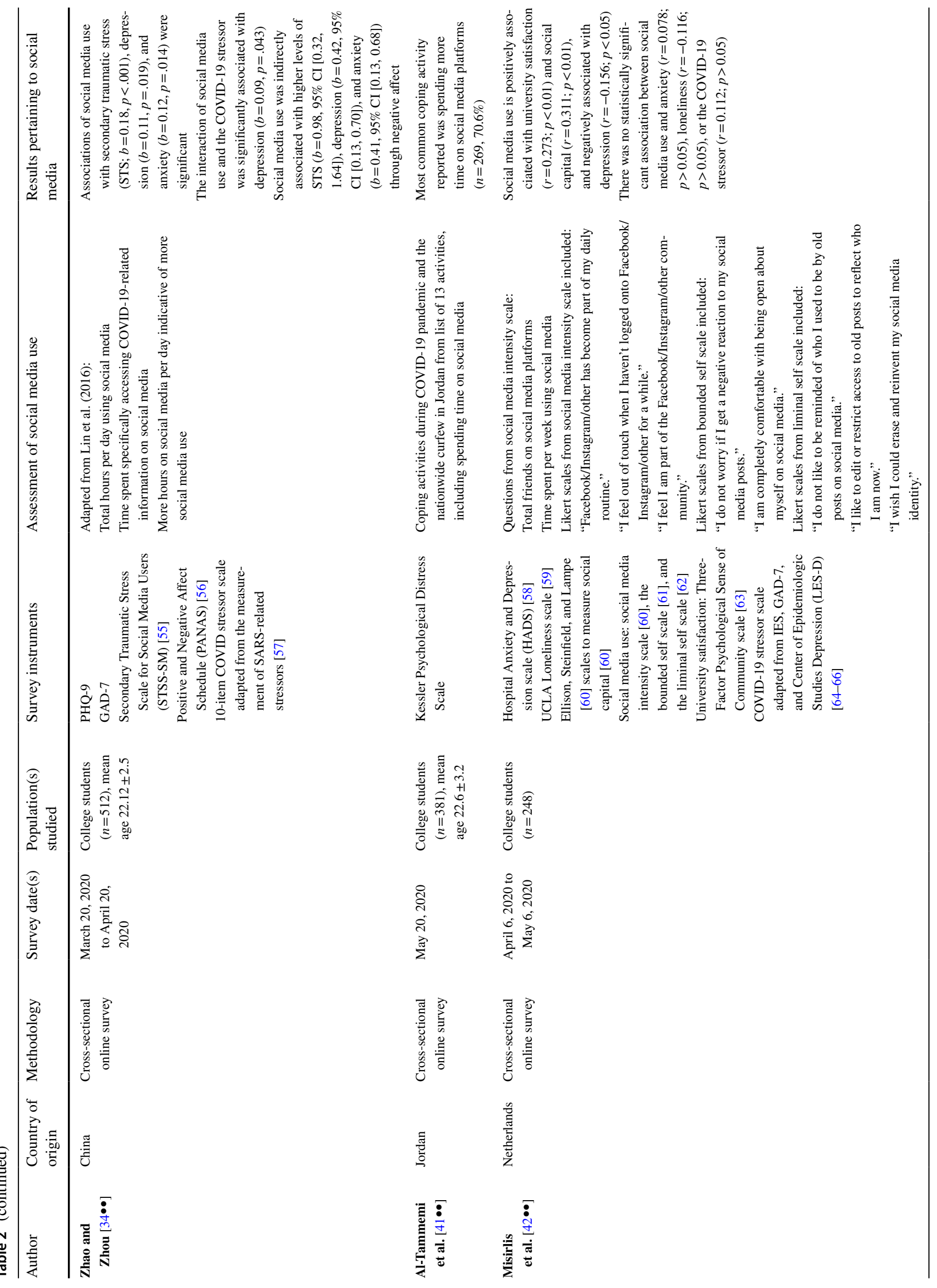


health through rumination. In fact, social media exposure was positively associated with rumination in students who had low levels of mindfulness; however, this relationship did not exist in students with high levels of mindfulness. The study authors concluded that students who utilized mindfulness more frequently were better able to regulate their intention and were less susceptible to ruminative thinking patterns. Thus, mindfulness may be a strategy to lessen the negative impact of social media on the mental health of students.

Another group in China [44•] explored the relationships between social media use, emotional regulation strategies, and overall mental health in the context of the COVID-19 pandemic. In particular, they focused on cognitive reappraisal (i.e., reinterpreting an emotion-provoking situation to reduce its emotional impact). Authors found that cognitive reappraisal was associated with increased positive mental health indicators (life satisfaction, adequacy) and decreased negative mental health indicators (depression). Interestingly, higher levels of cognitive reappraisal mitigated the adverse relationships between social media and mental health outcomes; among participants with high cognitive appraisal, the relationships disappeared between (1) judgement of others on social media and life satisfaction, (2) increased COVID19 online discussion and increased anxiety, and (3) sharing COVID-19 information and feelings of adequacy. Cognitive reappraisal might be a potential strategy to buffer the emotional charge of engaging with social media.

In summary, dialectical thinking (i.e., avoiding "all or nothing" thinking), optimism, mindfulness, and cognitive reappraisal are all possible strategies to mitigate the negative impact of social media on mental health during the COVID19 pandemic.

\section{Discussion}

\section{Summary of the Evidence}

The selected studies investigate the factors that affect the relationship between social media use and mental health among college students. Social media use appears to be associated with mental health symptoms, but the direction of these relations is mixed, likely due in part to moderating factors (e.g., excessive COVID-19 exposure, perceived social connectedness, and emotion-regulation strategies). The direction of causality of these associations cannot be determined with the current study designs as the large majority were cross-sectional; however, the authors hypothesize that college students are more reliant on social media because of the limitations in in-person interactions. Finally, these studies do not speak or explain the extent to which the pandemic may have strengthened the role that social media use might have on mental health. There are likely additional moderating variables that explain the relationships between the pandemic, social media, and college student mental health that were not accounted for in the included studies.

\section{Practicality of Social Media as a News Source}

While social media can serve as a tool for fostering connection during periods of physical isolation, the mental health implications of social media being used as a new source are tenuous. Several qualitative studies suggest that social media is most useful for rapidly disseminating timely information via widely accessible platforms. In the context of the COVID-19 pandemic, social media's prevalence worldwide was helpful in connecting users through existing networks. The ability to quickly communicate information about the pandemic was especially critical in the early months of the pandemic, as social media may have had instrumental value in rapid adaptation to sudden shifts in public health mandates. Information about COVID-19 has the potential both to empower and to overwhelm. Thus, social media's content may moderate the relations between social media use and mental health.

Vaccination is especially relevant today, and several recent studies have focused on social media as a news source of vaccine information. For example, a cross-sectional study of 6,922 Chinese college students investigated associations between intention to vaccinate and social media exposures [67]. Findings indicated that social media exposure is directly related to COVID vaccination intention, with both positive and negative associations depending on type of exposure. Future research should consider the influence of social media on public health issues, particularly in college students.

\section{Theoretical and Practical Implications}

\section{Defining Social Media}

The conceptualization of social media is challenged by the rate in which technology is expanding and evolving, such that new social media platforms are constantly being developed, modified, and abandoned across the globe. The most common social media services today include Facebook, YouTube, WhatsApp, Facebook Messenger, Instagram, WeChat, TikTok, QQ, Kakao, Douyin, Sina Weibo, Telegram, Snapchat, Kuaishou, Reddit, Pinterest, Twitter, and Quora [21]. Further complicating the terminology and definition, the acceleration of digital communication secondary to the COVID-19 pandemic has blurred the lines between social media services and other forms of technology used for communication purposes (e.g., e-mail, fax machine, and telephone). 
The functionality of social media is also variable. While social media was initially developed to facilitate inter-user connectivity, communication, and community-building, some users primarily use social media for consuming information and content discovery. In the context of pandemicrelated lockdowns, clinicians must consider whether social media is being used due to limited availability of alternative activities or as a primary coping strategy. Furthermore, there is currently no consensus on the definition for the term social media addiction. In the current Diagnostic and Statistical Manual of Mental Disorders (DSM), it is listed as a "condition for further study," and there is controversy as to whether excessive time spent on internet-based social media is an additive behavioral disorder. Developing standardized, universally accepted definitions for the terms social media, social media use, and social media addiction would increase the internal reliability and external validity of future research studies.

\section{Best Practices for Clinicians}

It is common to see a surge in social media usage after natural disasters as individuals are gathering information [68, 69]; anxiety levels tend to rise as individuals are inundated with information about the crisis on social media [68]. Thus, we would expect to see heightened anxiety in the setting of increased social media use by college students during the COVID-19 pandemic. However, given that smartphone usage is so ingrained into our daily lives and some social media use may facilitate supportive interpersonal relationships, completely abstaining from social media may be counter-productive. In fact, research has shown that positive usage of social media can be a form of psychological "first aid" in disastrous situations [69]. Therefore, the focus should be prevention of problematic social media use through moderation and active engagement rather than complete abstinence [70, 71]. Particularly, clinicians should be aware of social media's influence on college student mental health so that they can identify high-risk patients and guide them towards healthy social media behaviors. Clinicians should begin by asking college students to think deeply about their social media use. Then, there are a variety of screening tools designed to identify problematic social media use. The Young's Internet Addiction Test, Online Social Support Scale, Bergen Social Media Addiction Scale, Smartphone Addiction Scale, Internet Gaming Disorder Scale, and Instagram and Well-Being Questionnaire address the six domains of salience, impulsivity, mood modifications, tolerance withdrawal, and relapse to assess problematic social media use.

- Salience: Do you spend a lot of time thinking about social media or planning how to use it? [72]
- Impulsivity: Do you have a hard time concentrating in class, while doing assignments, or while working due to social media use? [73]

- Mood modifications: Do you use social media in order to temporarily escape or relieve a negative mood? [72, 74]

- Tolerance: Do you feel the need to spend increasing amounts of time engaged on social media in order to achieve satisfaction or pleasure? [75]

- Withdrawal: Do you feel more irritable, anxious, or sad when you are prohibited from using social media? [72, 75]

- Relapse: Have you repeatedly made unsuccessful efforts to control, cut back, or stop social media use? [76]

Anticipatory guidance during wellness visits with collegeaged patients, especially those that they identify as high-risk for problematic social media use, may include encouraging healthy habits, sleep hygiene, moderating social media use, active (rather than passive) engagement with social media, establishing media-free times during meals and studying, and privacy considerations $[77 \bullet]$.

\section{Future Directions}

While not the focus of the current article, it is crucial to note that social media use may present unique challenges for individuals with preexisting psychiatric disorders during the COVID-19 pandemic due to vulnerabilities associated with each disorder. As opposed to neurotypical individuals, neurodiverse individuals have a complex and heterogenous relationship with social media, especially in the setting of online learning [78]. For example, the characteristic impulsivity and distractibility associated with attention-deficit/ hyperactivity disorder (ADHD) may cause individuals to have trouble limiting time spent on social media, causing them to spend more time than desired on social networking sites at baseline; the pandemic may exacerbate these troubles and cause patients with ADHD to spend more time viewing pandemic-related content. For individuals with autism spectrum disorder (ASD), distance learning may eliminate social pressures that can feel overwhelming or distracting; however, the heterogeneity of skills, abilities, and needs of neurodiverse students can cause academic and personal difficulties [79]. In fact, symptoms of ADHD, ASD, major depressive disorder, and anxiety were shown to account for significant variance in problematic social media use in a large cross-sectional study of 16-88-year-olds prior to the pandemic [72]. Additionally, a cross-sectional study among college students prior to the COVID-19 pandemic showed that less frequent social media use was associated with reduced symptoms of depression [80], which needs to be interpreted cautiously in the setting of research showing 
many mediating and moderating factors affecting the relationship between social media use and both depression and anxiety $[81 \bullet, 82 \bullet, 83]$. Living in a COVID-19 pandemic world may increase the amount of time spent online, making this relationship stronger.

The rapid evolution of social media platforms-and changes in users' intentions when seeking them out-also merits further consideration, given that shifts in social usage may have unique psychological ramifications. For example, using social media as a news source may have implications for anxiety, particularly during the COVID-19 pandemic, when inflammatory content and misinformation circulated widely on these platforms. Several qualitative studies [84•, $85 \bullet, 86]$ not discussed in this paper suggested that social media is useful for rapidly and broadly disseminating timely information, but noted that rampant misinformation on these platforms contributed to confusion and stress throughout the COVID-19 pandemic. Future research might parse the nuances of how and why college users engage with particular forms of social media (including relying on it as a news source) and describe the psychological effects of each. These findings may contribute to the creation of a comprehensive, standard definition of social media.

While most of the scientific literature focusing on the relationship between social media use and mental health outcomes was prior to the COVID-19 pandemic, it is reasonable to infer that these relationships remain consistent, if not stronger, during a time where social media is more heavily relied on for all types of communication. Additionally, integrating previous literature with new findings during the pandemic may lead to interesting new insights. For example, one pre-pandemic study found that using social media to relieve boredom was correlated to anxiety 3 years later; however, using social media for information gathering was not [82•]. As discussed previously, the high COVID-19 stressor changes the relationship between high social media

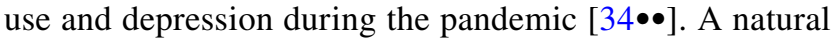
question then emerges: how do these entities integrate? Do stressors related to disasters become a mediating factor that was not considered prior to the COVID-19 pandemic? Or do our new findings seamlessly integrate so that using social media to relieve boredom leads to more anxiety in the setting of high COVID-19 stressors? More research will be needed to elucidate these new relationships.

\section{Limitations}

The studies selected for this review were conducted during a global pandemic in which new issues and challenges were frequently emerging, limiting the repository of available data. Furthermore, most of our selected articles were based out of China and may not be generalizable to the global population. Due to strict eligibility criteria, there is a possibility that we failed to identify studies relevant to the mental health and social media use of college students. Our conclusions and recommendations are therefore based on limited data, which calls for caution with interpreting the results. Future literature reviews utilizing larger datasets are required to confirm our findings.

\section{Conclusion}

This literature review explored the impact of social media use on college student mental health during the COVID-19 pandemic. Social media use has increased among almost all cohorts during the COVID-19 era, but college students have been especially affected. The pandemic made internet use nonnegotiable. College students had both positive and negative experiences online depending on a variety of factors. We found that excessive or problematic social media use was correlated with worse mental health outcomes overall, especially depression. COVID-19 acts as a moderator by strengthening the relationship between social media use and mental health. Dialectical thinking, optimism, mindfulness, and cognitive reappraisal are all possible strategies to mitigate the negative impact of social media on mental health during the COVID-19 pandemic. Clinicians should ask college students about their social media use to screen for associated mental health issues and to promote healthy internet use. The paucity of literature available about this topic limits the generalizability of this review. Future studies should consider the impact of social media on college student mental health and concentrate on intervention initiatives to ensure the psychological well-being of college students during a global pandemic.

\section{Compliance with Ethical Standards}

Conflict of Interest The authors declare no competing interests.

Human and Animal Rights and Informed Consent This article does not contain any studies with human or animal subjects performed by any of the authors.

\section{References}

Papers of particular interest, published recently, have been highlighted as:

- Of importance

$\bullet$ Of major importance

1. da Silva ML, Rocha RSB, Buheji M, Jahrami H, Cunha KDC. A systematic review of the prevalence of 
anxiety symptoms during coronavirus epidemics. J Health Psychol. 2020;71:135910532095162-11. https://doi.org/10.1177/13591 05320951620.

2. Loades ME, Chatburn E, Higson-Sweeney N, Reynolds S, Shafran R, Brigden A, et al. Rapid systematic review: the impact of social isolation and loneliness on the mental health of children and adolescents in the context of COVID-19. J Am Acad Child Adolesc Psychiatry. 2020; 1-46. https://doi.org/ 10.1016/j.jaac.2020.05.009.

3. Luo M, Guo L, Yu M, Jiang W, Wang H. The psychological and mental impact of coronavirus disease 2019 (COVID-19) on medical staff and general public - a systematic review and metaanalysis. Psychiatry Research Elsevier Ireland Ltd. 2020;291: 113190. https://doi.org/10.1016/j.psychres.2020.113190.

4. Machado DB, Alves FJO1, Teixeira CSS, Rocha AS, Castro-de-Araujo LF, Singh A, et al. Effects of COVID-19 on anxiety, depression and other mental health issues: a worldwide scope review. ResearchSquare. 2020. doi: https://doi.org/10.21203/rs.3.rs-58186/v1.

5. Vindegaard N, Benros ME. COVID-19 pandemic and mental health consequences: systematic review of the current evidence. Brain, Behavior, and Immunity. Academic Press. 2020. https://doi.org/10.1016/j.bbi.2020.05.048.

6. Aristovnik A, Keržič D, Ravšelj D, Tomaževič N, Umek L. Impacts of the COVID-19 pandemic on life of higher education students: a global perspective. Sustainability. 2020;12(20):8438.

7. Browning MHEM, Larson LR, Sharaievska I, Rigolon A, McAnirlin O, Mullenbach L, et al. Psychological impacts from COVID-19 among university students: risk factors across seven states in the United States. PLoS One. 2021;16(1):e0245327. https://doi.org/10.1371/journal.pone.0245327.

8. Li S, Wang Y, Yang Y, Lei X, Yang Y. Analysis of influencing factors of anxiety and emotional disorders in children and adolescents during home isolation during the epidemic of novel coronavirus pneumonia. Chin. J. Child Heal. 2020; 1-9.

9.•• Household Pulse Survey. U.S. Census Bureau. 2020 [updated 2020 Dec 21; cited 2021 Jan 3]. Available from: https://www. census.gov/programs-surveys/household-pulse-survey/data. $\mathrm{html}$. This dataset measures household experiences during the coronavirus pandemic at the national and state levels, providing valuable insight on social and economic trends.

10. Aylie NS, Mekonen MA, Mekuria RM. The psychological impacts of COVID-19 pandemic among university students in Bench-Sheko zone, South-west Ethiopia: a communitybased cross-sectional study. Psychol Res Behav Manag. 2020;13:813-21. https://doi.org/10.2147/PRBM.S275593.

11. Gritsenko V, Skugarevsky O, Konstantinov V, Khamenka N, Marinova T, Reznik A, Isralowitz R. COVID 19 fear, stress, anxiety, and substance use among Russian and Belarusian university students. Int J Ment Heal Addict. 2020; 1-7. Advance online publication. https://doi.org/10.1007/s11469-020-00330-z.

12. Husky MM, Kovess-Masfety V, Swendsen JD. Stress and anxiety among university students in France during Covid-19 mandatory confinement. Compr Psychiatry. 2020;102: 152191. https://doi.org/10.1016/j.comppsych.2020.152191.

13. Kaparounaki CK, Patsali ME, Mousa DV, Papadopoulou E, Papadopoulou K, Fountoulakis KN. University students' mental health amidst the COVID-19 quarantine in Greece. Psychiatry Res. 2020;290: 113111. https://doi.org/10.1016/j.psych res.2020.113111.

14. Son C, Hegde S, Smith A, Wang X, Sasangohar F. Effects of COVID-19 on college students' mental health in the United States: interview survey study. J Med Internet Res. 2020;22(9): e21279. https://doi.org/10.2196/21279.

15. Tasnim R, Islam MS, Sujan M, Sikder MT, Potenza MN. Suicidal ideation among Bangladeshi university students early during the COVID-19 pandemic: prevalence estimates and correlates. Child Youth Serv Rev. 2020;119: 105703. https:// doi.org/10.1016/j.childyouth.2020.105703.

16. Wang X, Hegde S, Son C, Keller B, Smith A, Sasangohar F. Investigating mental health of US college students during the COVID-19 pandemic: cross-sectional survey study. J Med Internet Res. 2020;22(9): e22817. https://doi.org/10.2196/22817.

17. Wathelet M, Duhem S, Vaiva G, Baubet T, Habran E, Veerapa E, et al. Factors associated with mental health disorders among university students in France confined during the COVID-19 pandemic. JAMA Netw Open. 2020;3(10): e2025591. https:// doi.org/10.1001/jamanetworkopen.2020.25591.

18. Conrad RC, Koire A, Pinder-Amaker S, Liu CH. College student mental health risks during the COVID-19 pandemic: implications of campus relocation. J Psychiatr Res. 2021;136:117-26.

19. Liu CH, Pinder-Amaker S, Hahm HC, Chen JA. Priorities for addressing the impact of the COVID-19 pandemic on college student mental health. J Am Coll Heal. 2020;1-3.

20. Sankhi S, Marasine NR. Impact of COVID-19 pandemic on mental health of the general population, students, and health care workers. Europasian J Med Sci. 2020;2(Covid-19 Special Issue):1-9. https://doi.org/10.46405/ejms.v2i2.131.

21. Kemp S. More than half of the people on Earth now use social media. DataReportal. 2020. https://datareportal.com/reports/ more-than-half-the-world-now-uses-social-media. Accessed 4 Jan 2021.

22. Elhai JD, Yang H, McKay D, Asmundson GJG. COVID-19 anxiety symptoms associated with problematic smartphone use severity in Chinese adults. J Affect Disord. Elsevier B.V; 2020; 274:576-582. https://doi.org/10.1016/j.jad.2020.05.080.

23. Stieger S, Lewetz D, Swami V. Psychological well-being under conditions of lockdown: an experience sampling study in Austria during the COVID-19 pandemic. 2020. https://doi.org/10.31234/ osf.io/qjhfp.

24.• Gómez-Galán J, Martínez-López JÁ, Lázaro-Pérez C, Sarasola Sánchez-Serrano JL. Social networks consumption and addiction in college students during the COVID-19 pandemic: educational approach to responsible use. Sustainability. 2020; 12:7737. This Spanish cross-sectional study demonstrated increased rates of social media addiction among college students during the COVID pandemic.

25. Majumdar P, Biswas A, Sahu S. COVID-19 pandemic and lockdown: cause of sleep disruption, depression, somatic pain, and increased screen exposure of office workers and students of India. Chronobiol Int. 2020;37(8):1191-200. https://doi.org/10. 1080/07420528.2020.1786107.

26. Rajkumar RP. COVID-19 and mental health: a review of the existing literature. Asian J Psychiatr. 2020;52:102066. https:// doi.org/10.1016/j.ajp.2020.102066. This article is one of the first literature reviews of mental health during the COVID pandemic.

27. Twenge JM, Haidt J, Joiner TE, Campbell WK. Underestimating digital media harm. Nat Hum Behav. 2020;4:346-8. https://doi. org/10.1038/s41562-020-0839-4.

28.• Gao J, Zheng P, Jia Y, Chen H, Mao Y, Chen S, Wang Y, Fu H, Dai J. Mental health problems and social media exposure during COVID-19 outbreak. PLoS One. 2020;15(4):e0231924.

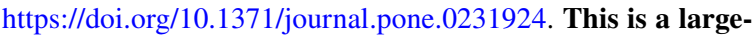
scale cross-sectional study from China that found a positive association between social media exposure and rates of anxiety and depression in the general population.

29. Zhong B, Huang Y, Liu Q. Mental health toll from the coronavirus: social media usage reveals Wuhan residents' depression and secondary trauma in the COVID-19 outbreak. Comput Human Behav. 2021;114:106524. https://doi.org/10.1016/j.chb.2020. 106524. This Chinese cross-sectional study administered at the peak of the COVID-19 outbreak showed that social media 
usage predicted both depression and secondary trauma in Wuhan residents.

30. Huckins JF, daSilva AW, Wang W, Hedlund E, Rogers C, Nepal SK, et al. Mental health and behavior of college students during the early phases of the COVID-19 pandemic: longitudinal smartphone and ecological momentary assessment study. J Med Internet Res. 2020;22: e20185. https://doi.org/10.2196/20185.

31. Sundarasen S, Chinna K, Kamaludin K, Nurunnabi M, Baloch GM, Khoshaim HB, et al. Psychological impact of COVID-19 and lockdown among university students in Malaysia: implications and policy. Recommendations IJERPH. 2020;17:6206-13. https://doi.org/10.3390/ijerph17176206.

32. O'Brien M, Moore K, McNicholas F. Social media spread during Covid-19: the pros and cons of likes and shares. Ir Med J. 2020;113(4):52. This editorial piece lays out the pros and cons of social media use specifically during the COVID pandemic.

33. Pahayahay A, Khalili-Mahani N. What media helps, what media hurts: a mixed methods survey study of coping with COVID-19 using the media repertoire framework and the appraisal theory of stress. J Med Internet Res. 2020;22(8):e20186. https://doi.org/ 10.2196/20186. This American survey study demonstrates an increase in social media use as a coping mechanism for COVID-related stress.

34.•• Zhao N, Zhou G. Social media use and mental health during the COVID-19 pandemic: moderator role of disaster stressor and mediator role of negative affect. Appl Psychol Health Well Being. 2020;12(4):1019-38. https://doi.org/10.1111/aphw. 12226. This is an original observational study of college mental health related to social media use during the COVID-19 pandemic.

35.•• Allcott H, Braghieri L, Eichmeyer S, Gentzkow M. The welfare effects of social media (No. w25514). Am Econ Rev. 2019;110(3):629-76. This randomized controlled study showed that decreasing Facebook use leads to increases in subjective well-being while also decreasing factual knowledge and polarization.

36. World Health Organisation. Communicating risk in public health emergencies [Internet]. Geneva: World Health Organisation; 2017. Available from: https://apps.who.int/iris/bitstream/handle/10665/ 259807/9789241550208-eng.pdf?sequence $=2$. Accessed 3 Jan 2021.

37. Naeem M. Do social media platforms develop consumer panic buying during the fear of Covid-19 pandemic. J Retail Consum Serv. 2021;58: 102226. https://doi.org/10.1016/j.jretconser.2020.102226.

38.• Li M, Liu L, Yang Y, Wang Y, Yang X, Wu H. Psychological impact of health risk communication and social media on college students during the COVID-19 pandemic: cross-sectional study. J Med Internet Res. 2020;22(11):e20656. https://doi.org/ $10.2196 / 20656$. This is an original observational study of college mental health related to social media use during the COVID-19 pandemic.

39.•• Li Y, Zhao J, Ma Z, McReynolds LS, Lin D, Chen Z, Wang T, Wang D, Zhang Y, Zhang J, Fan F, Liu X. Mental health among college students during the COVID-19 pandemic in China: a 2-wave longitudinal survey. J Affect Disord. 2020;281:597-604. https://doi.org/10.1016/j.jad.2020.11.109. This is an original observational study of college mental health related to social media use during the COVID-19 pandemic.

40.• Hong W, Liu RD, Ding Y, Fu X, Zhen R, Sheng X. Social media exposure and college students' mental health during the outbreak of COVID-19: the mediating role of rumination and the moderating role of mindfulness. Cyberpsychol Behav Soc Netw. 2020. https://doi.org/10.1089/cyber.2020. 0387. Advance online publication. https://doi.org/10.1089/ cyber.2020.0387. This is an original observational study of college mental health related to social media use during the COVID-19 pandemic.

41.• Al-Tammemi AB, Akour A, Alfalah L. Is it just about physical health? An online cross-sectional study exploring the psychological distress among university students in Jordan in the midst of COVID-19 pandemic. Front Psychol. 2020;11:562213. https://doi.org/10.3389/fpsyg.2020.562213. (This is an original observational study of college mental health related to social media use during the COVID-19 pandemic.)

42.•• Misirlis N, Zwaan MH, Weber D. International students' loneliness, depression and stress levels in COVID-19 crisis: the role of social media and host university. arXiv. 2020. Available online: https://arxiv.org/abs/2005.12806. Accessed 20 Dec 2020. This is an original observational study of college mental health related to social media use during the COVID-19 pandemic.

43. Giusti L, Salza A, Mammarella S, Bianco D, Ussorio D, Casacchia M, Roncone R. \#Everything will be fine. Duration of home confinement and "all-or-nothing" cognitive thinking style as predictors of traumatic distress in young university students on a digital platform during the COVID-19 Italian lockdown. Front Psych. 2020;11:574812. https://doi.org/10.3389/fpsyt.2020.574812. This cross-sectional study explores how different thinking styles predicted posttraumatic symptomatology.

44. Yang Y, Liu K, Li S, Shu M. Social media activities, emotion regulation strategies, and their interactions on people's mental health in COVID-19 pandemic. Int J Environ Res Public Health. 2020;17(23), 8931. https://doi.org/10.3390/ ijerph17238931. This article explains how emotion-regulation strategies mediate the relationship between social media use and mental health.

45. Derogatis L. SCL-90-R: administration, scoring, and procedures manual. Minneapolis, MN: NCS Pearson; 1996.

46. Salkovskis PM, Rimes KA, Warwick HMC, Clark DM. The Health Anxiety Inventory: development and validation of scales for the measurement of health anxiety and hypochondriasis. Psychol Med. 2002;32(5):843-53. https://doi.org/10.1017/s0033 291702005822 .

47. Kroenke K, Spitzer RL, Williams JB. The PHQ-9: validity of a brief depression severity measure. J Gen Intern Med. 2001;16(9):606-13.

48. Spitzer RL, Kroenke K, Williams JB, Löwe B. A brief measure for assessing generalized anxiety disorder: the GAD-7. Arch Intern Med. 2006;166(10):1092-7.

49. Thoresen S, Tambs K, Hussain A, Heir T, Johansen VA, Bisson JI. Brief measure of posttraumatic stress reactions: impact of event scale-6. Soc Psychiatry Psychiatr Epidemiol. 2010;45(3):405-12. https://doi.org/10.1007/s00127-009-0073-x.

50. Weiss DS. The impact of event scale: revised. In Wilson JP, Tang CS-K (eds.). International and cultural psychology. Crosscultural assessment of psychological trauma and PTSD. Springer Science + Business Media. 2007; p. 219-238. https://doi.org/10. 1007/978-0-387-70990-1_10.

51. Greco LA, Baer RA, Smith GT. Assessing mindfulness in children and adolescents: development and validation of the Child and Adolescent Mindfulness Measure (CAMM). Psychol Assess. 2011;23:606-14.

52. Liu X, Chi X, Zhang J, et al. Validation of child and adolescent mindfulness measure (CAMM) in Chinese adolescents [in Chinese]. Psychol Explor. 2019;39:250-6.

53. Zhou X, Wu X. The relationship between rumination, posttraumatic stress disorder, and posttraumatic growth among Chinese adolescents after earthquake: a longitudinal study. J Affect Disord. 2016;193:242-8. https://doi.org/10.1016/j.jad. 2015.12.076.

54. Kessler RC, Andrews G, Colpe LJ, Hiripi E, Mroczek DK, Normand SLT, et al. Short screening scales to monitor population prevalences 
and trends in non-specific psychological distress. Psychol Med. 2002;32:959-76. https://doi.org/10.1017/S0033291702006074.

55. Bride BE, Robinson MM, Yegidis B, Figley CR. Development and validation of the secondary traumatic stress scale. Res Soc Work Pract. 2004;14(1):27-35.

56. Watson D, Clark LA, Tellegen A. Development and validation of brief measures of positive and negative affect: the PANAS scales. J Pers Soc Psychol. 1988;54(6):1063-70.

57. Main A, Zhou Q, Ma Y, Luecken LJ, Liu X. Relations of SARSrelated stressors and coping to Chinese college students' psychological adjustment during the 2003 Beijing SARS epidemic. J Couns Psychol. 2011;58(3):410-23.

58. Zigmond AS, Snaith RP. Hospital anxiety and depression scale. Acta Psychiatr Scand. 1983;67(6):361-70. https://doi.org/10. 1111/j.1600-0447.1983.tb09716.x.

59. Russell D, Peplau LA, Cutrona CE. The revised UCLA loneliness scale: concurrent and discriminant validity evidence. J Pers Soc Psychol. 1980;39(3):472.

60. Ellison NB, Steinfield C, Lampe C. The benefits of Facebook "friends:" social capital and college students' use of online social network sites. J Comput-Mediat Commun. 2007;12(4):1143-68. https://doi.org/10.1111/j.1083-6101.2007.00367.x.

61. Boyd DM, Ellison NB. Social network sites: definition, history, and scholarship. J Comput-Mediat Commun. 2007;13(1):210-30.

62. Kerrigan F, Hart A. Theorising digital personhood: a dramaturgical approach. J Mark Manag. 2016;32(17-18):1701-21.

63. Jason LA, Stevens E, Ram D. Development of a three-factor psychological sense of community scale. J Community Psychol. 2015;43(8):973-85.

64. Eaton WW, Smith C, Ybarra M, Muntaner C, Tien A. Center for epidemiologic studies depression scale: review and revision (CESD and CESD-R). In Maruish ME (ed.), The use of psychological testing for treatment planning and outcomes assessment: Instruments for adults. Lawrence Erlbaum Associates Publishers; 2004. p. 363-377.

65. Radloff LS. The use of the Center for Epidemiologic Studies Depression Scale in adolescents and young adults. J Youth Adolesc. 1991;20(2):149-66.

66. Roberts RE, Andrews JA, Lewinsohn PM, Hops H. Assessment of depression in adolescents using the Center for Epidemiologic Studies Depression Scale. J Consult Clin Psychol. 1990;2(2):122.

67. Luo S, Xin M, Wang S, Zhao J, Zhang G, Li L, Li L, Tak-fai LJ. Behavioural intention of receiving COVID-19 vaccination, social media exposures and peer discussions in China. Epidemiol Infect. 2021;149(e158):1-9.

68. Saroj A, Pal S. Use of social media in crisis management: a survey. Int J Disaster Risk Reduct. 2020;48:101584.

69. Taylor M, Wells G, Howell G, Raphael B. The role of social media as psychological first aid as a support to community resilience building. A Facebook study from 'Cyclone Yasi Update.' Aust J Emerg Manag. 2012;27(1):20-6.

70. Kuss D, Griffiths M. Social networking sites and addiction: ten lessons learned. Int J Environ Res Public Health. 2017;14(3):311.

71. Lin L, Sidani J, Shensa A, Radovic A, Miller E, Colditz J, Hoffman B, Giles L, Primack B. Association between social media use and depression among US young adults. Depress Anxiety. 2016;33(4):323-31.

72. Andreassen CS, Billieux J, Griffiths MD, Kuss DJ, Demetrovics $\mathrm{Z}$, Mazzoni E, Pallesen S. The relationship between addictive use of social media and video games and symptoms of psychiatric disorders: a large-scale cross-sectional study. Psychol Addict Behav. 2016;30(2):252.

73. Kwon M, Kim DJ, Cho H, Yang S. The smartphone addiction scale: development and validation of a short version for adolescents. PLoS One. 2013;8(12): e83558. https://doi.org/10.1371/ journal.pone. 0083558 .
74. Csibi S, Griffiths M, Cook B, Demetrovics Z, Szabo A. The psychometric properties of the smartphone applicationbased addiction scale (SABAS). Int J Ment Heal Addict. 2017;16(2):393-403.

75. Pontes H, Griffiths M. Measuring DSM-5 internet gaming disorder: development and validation of a short psychometric scale. Comput Hum Behav. 2015;45:137-43. https://doi.org/10.1016/j. chb.2014.12.006.

76. Young KS. Caught in the net: how to recognize the signs of internet addiction - and a winning strategy for recovery. New York: Wiley; 1998.

77. Hill D. Social media: anticipatory guidance. Pediatr Rev. 2020;41(3):112-9. https://doi.org/10.1542/pir.2018-0236. This review article in the American Academy of Pediatrics is about how and why clinicians should provide anticipatory guidance to children/adolescents and their families regarding healthy social media use.

78. Rowello L. "Neurodivergent people share their experience of the pandemic." How Did the Pandemic Affect Neurodivergent Individuals?, Verywell Mind, 14 June 2021, https://www.verywellmind.com/howneurodivergence-impacts-experiences-of-the-pandemic-5186107.

79. Adams D, Simpson K, Davies L, Campbell C, Macdonald L. Online learning for university students on the autism spectrum: a systematic review and questionnaire study. Australas J Educ Technol. 2019;35(6).

80. Ghaemi SN. Digital depression: a new disease of the millennium? Acta Psychiatr Scand. 2020;141(4):356-61.

81. Sarmiento IG, Olson C, Yeo G, et al. How does social media use relate to adolescents' internalizing symptoms? Conclusions from a systematic narrative review. Adolescent Res Rev. 2020;5:381-404. This systematic review demonstrates the mental health benefits of actively rather than passively engaging with social media.

82. Stockdale LA, Coyne SM. Bored and online: reasons for using social media, problematic social networking site use, and behavioral outcomes across the transition from adolescence to emerging adulthood. J Adolesc. 2020;79:173-183. This longitudinal study explores how motivations for using social networking sites relate to mental health outcomes.

83. Thorisdottir IE, Sigurvinsdottir R, Asgeirsdottir BB, Allegrante JP, Sigfusdottir ID. Active and passive social media use and symptoms of anxiety and depressed mood among Icelandic adolescents. Cyberpsychol Behav Soc Netw. 2019;22(8):535-42.

84. Jayaseelan R, Brindha D, Kades W. Social media reigned by information or misinformation about COVID-19: a phenomenological study. SSRN Electron J. 2020. https://doi.org/10.2139/ ssrn.3596058. This qualitative study explores whether social media is diffusing information or misinformation to the public about the COVID-19 pandemic.

85. Lin CY, Broström A, Griffiths MD, Pakpour AH. Investigating mediated effects of fear of COVID-19 and COVID-19 misunderstanding in the association between problematic social media use, psychological distress, and insomnia. Internet Interv. 2020;21:100345. https://doi.org/10.1016/j.invent.2020. 100345. This survey found a correlation between high intake of COVID-19-related misinformation with psychological distress, particularly in the young adult population.

86. Suhail A, Iqbal N, Smith J. Lived experiences of Indian youth amid COVID-19 crisis: an interpretative phenomenological analysis. International J Soc Psychiatry. 20764020966021. 2020. Advance online publication. https://doi.org/10.1177/ 0020764020966021.

Publisher's Note Springer Nature remains neutral with regard to jurisdictional claims in published maps and institutional affiliations. 